\title{
WitMirror: A Voice Controlled IOT Mirror
}

\author{
Abdul Ahad ${ }^{1}$, Kiran Panwar ${ }^{2}$, Vivek Arya ${ }^{3}$, Vinayak Lohan ${ }^{4}$ and Purnendu Shekhar \\ Pandey ${ }^{5}$ \\ 1,2,3,4 B.Tech Student, ${ }^{5}$ Assistant Professor, Department of Electronics \& \\ Communication Engineering, THDC-IHET, Bhagirathipuram, Tehri Garhwal, \\ Uttrakhand-249124, India \\ 1abduls060@gmail.com
}

\begin{abstract}
It is a self-evident fact that modern technology is transforming everything around us. With the advent of IoT era, this transformation is even more remarkable. From our phones to even our shoes, everything is becoming smarter. Even the most ubiquitous thing, like a mirror, is no exception. Through this paper we have explained how even a simple looking mirror can interact with us, providing us with much useful information and giving us the ease of controlling each and everything around us, just by using voice commands. This paper depicts the workflow and development of WitMirror. As the name suggests, this device has the ability to interact with the user through voice commands giving the output accordingly. It can display various useful information like current weather, date \& time, news headlines, map of the desired location and all the upcoming events. For extracting these piece of information, the device will use various third-party APIs through the Internet. It is the next step in the field of home automation, where the mirror acts as a central hub, which can control all the home devices dwelling in the smart futuristic home. For parents, it also has a unique application where they can monitor the current location of their children through a simple android app installed on their phone. So in case of any miss happening, the parents will know the location of their children beforehand. The user face will be detected using image processing. Raspberry Pi 3 will work as the brain of the device. It is also equipped with a microphone, camera speaker and a display for various input/output purpose. For controlling Home Appliances relay mechanism is used. With all these advanced features, the device will also act as the simple mirror for astounding experience to the user.
\end{abstract}

Keywords: Internet of Things (IoT), Home automation, Raspberry pi

\section{Introduction}

Every invention aims to make the life easier. We are all aware of the importance of time in this modern era. A person on an average spends 2 weeks per year in front of a mirror. Moreover, on daily basis, we spend our 10-15 minutes in front of a mirror just to select the suitable outfit or a look for the day [1]. It will be very beneficial if that time is utilized in some productive ways. Furthermore, that time utilization with astounding experience will be the icing on the cake. This paper has been prepared in the same context of time utilization in an enjoyable manner.

Article history:

Received (April 16, 2019), Review Result (May 26, 2019), Accepted (July 18, 2019) 
We all know how common a mirror is. It is just a reflecting surface found mostly in every home. But what if we combine this simple looking reflecting surface with modern technology. The necessity of a mirror, in every home, makes it more susceptible to advancement than any other home item. So this amalgamation of a simple mirror with technology is our WitMirror. It is a simple two-way mirror with a display behind it which is used to show various data. A twoway mirror is partially reflected and partially transparent mirror. When there is enough light on one side of the mirror, light can pass through that side. On the darker side of the mirror, a person can see its own reflection, thus mimicking the device as a usual mirror.

The main controlling device for WitMirror is Raspberry Pi 3. It acts as its brain and does all the required computations. For operating the Pi machine, the recommended Raspbian Operating System is used, which provides the stable and fast environment for the device. Being Debian based operating system, Raspbian is Open-Source and thus free and customizable. All the programming is done in Python language because of its easy syntax the presence of various speech and image processing modules.

The basic structural block diagram of the project is shown in [Figure 1].

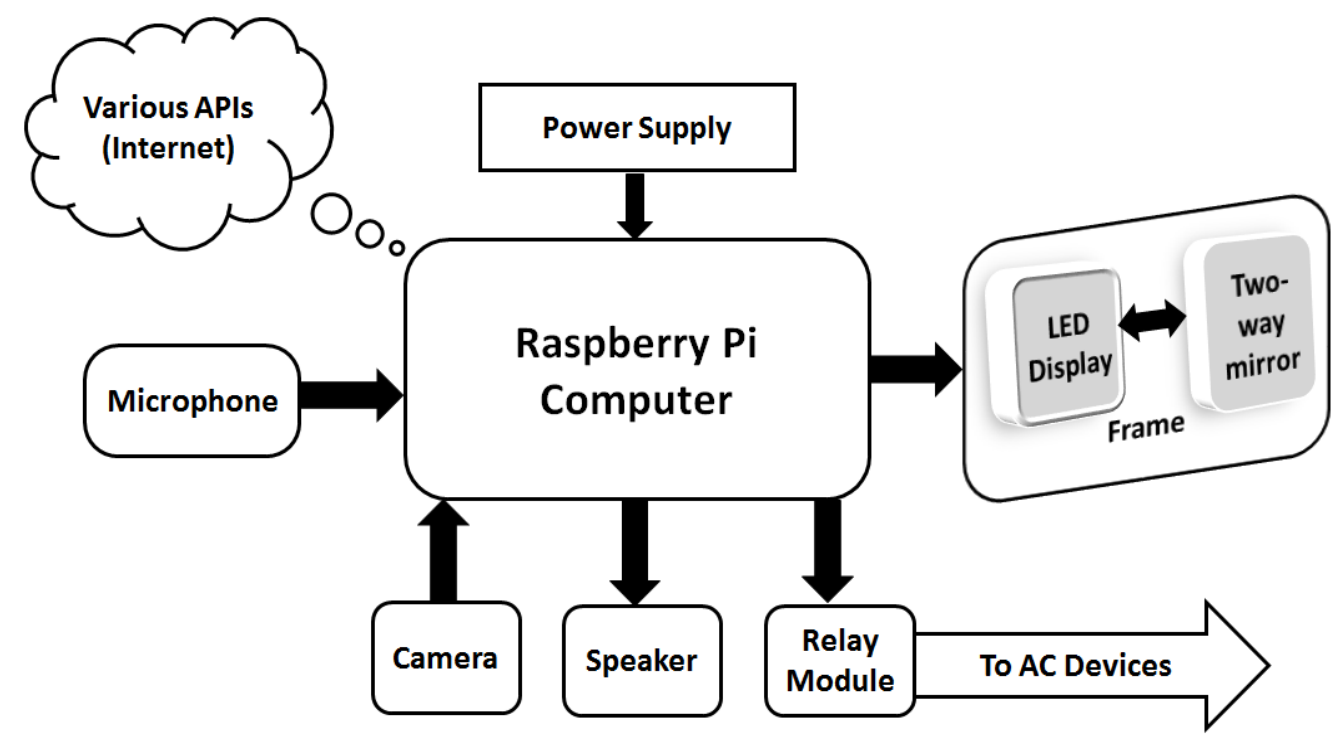

Figure 1. A structural block diagram showing the hardware components

The fact that the device can be controlled through voice commands makes it more appealing to the users. Even the physically challenged people can access the home devices by giving commands to the mirror. In return, the mirror can give answers as speech output which can further be beneficial for the blind people. Thus the device can also be worthwhile for elder and differentially abled people.

The rest of the paper is organized as follows. Section 2 gives the literature review or the related work, done previously on this topic. Section 3 describes the methodology used for the work. It includes planning, hardware \& software development including workflow of the project. After that, the study is concluded while commenting on its future scope in Section 4.

\section{Literature review}

As IoT is a booming technology, there are many people who have conducted researches on this topic. So before starting this research, it was necessary for us to investigate the previous 
work done related to this topic. Thousands of researchers have already used a two-way mirror for designing similar devices. So in this section, we will review the earlier researchers and projects in this direction.

One of the popular Interactive mirrors was created by Alpay Kasal and Sam Ewen of Lit Studios [2]. This mirror can be interacted using touch and gestures. It only has one point of touch as it is just imitating the mouse. This was just made for the entertainment purposes and did not intend to display the important data.

A group of five students at the Chalmers University of Technology in Sweden created a mirror called HUD mirror [3], with LEDs mounted behind the two-way mirror. These LEDs are used to show the information on the mirror by illuminating in a specific pattern. It displays the very specific amount of data like time, weather, temperature, etc. For interaction, this mirror uses LDR sensors and perform computations in Arduino. This mirror lacks flexibility and cannot display much useful information like maps.

Next project is the voice recognition based magic mirror created by the New York Times Research and Development Lab [4]. It tracks the movement using a Microsoft Kinect and runs completely on Windows. It uses the TV for the display and RFID reader for the identification of various bathroom items. It has one allured feature in which it "virtually" put clothes on users. It is integrated with social media and e-mail services. As this mirror works on Windows, it cannot be open source.

Next project is a simple smart mirror which displays limited data like date, time, weather and news feeds [5] This mirror simply displays information from the internet and does not have speech recognition and facial detection features. This mirror displays the picture of the women before and after putting the makeup.

Next example is of an augmentation mirror developed by Iwabuchi et al. [6]. It is a makeup mirror designed only for women. This mirror uses a camera of 1624 x 1224 resolution and helps women to put makeup by taking their pictures and displaying them. In all these five projects, we can see that how this device is different in each version. Initially, it was only able to display some information, then voice commands are integrated and at last it was equipped with the touchscreen. Still, there were some innovations left and there was the need to develop further interactivity. In [7][8] authors have reported design of mirrors to improve the productivity by utilizing the time, however failed to justify the design. In [2][9][10] authors reported entire setup of various utility places like bathroom, house and furniture for utilizing time and other aspects of the new mirroring technology. In [11] smart reflect has been proposed and tried to justify it and using the mirror effect for better time utilization and makeovers. In [11][12][13] authors have used the new technics of internet of things and the concepts of wireless technology for using such technology remotely and managing the households. In [14][15][16] authors have proposed many new concepts for better utilization of the technology and full utilization of the time.

\section{Methodology}

In this section, each phase of the development of this device is explained. It includes all the hardware and software requirements with the basic workflow of the design.

\subsection{Planning}

Before getting our hands dirty on some real implementation of this device, we first prepared our project plan by jotting down every hardware and software required for the device. Next, we planned about the time duration required for each phase followed by the budget planning. 
Finally, we analyzed the feasibility of the project to determine whether the implementation is technically feasible and economically profitable or not.

\subsection{Hardware development}

Implementation of the above plan, for development of the final working hardware, comes in this phase. It includes hardware description of the project.

The main hardware components are:

1) Raspberry Pi 3 B

2) LED display

3) Two-way mirror glass

4) Camera

5) Speaker

6) Microphone

7) Relay module

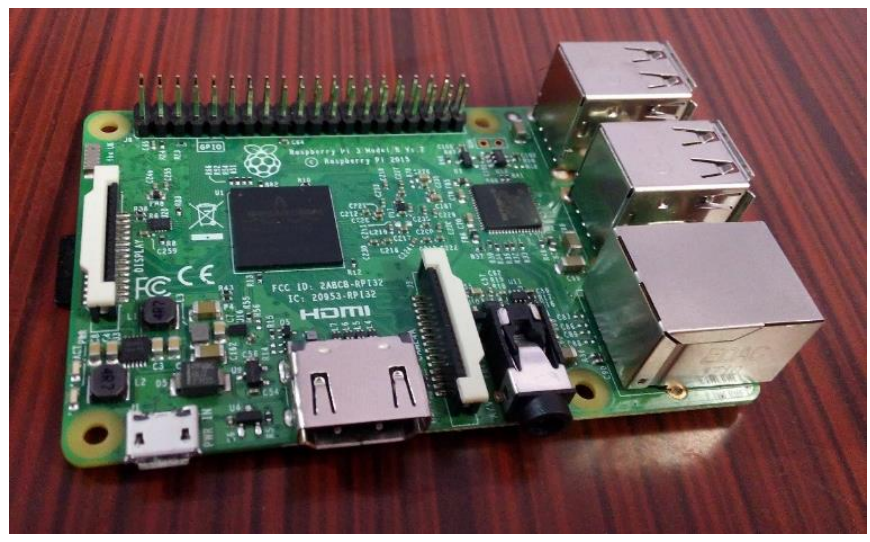

Figure 2. Raspberry pi model 3 B

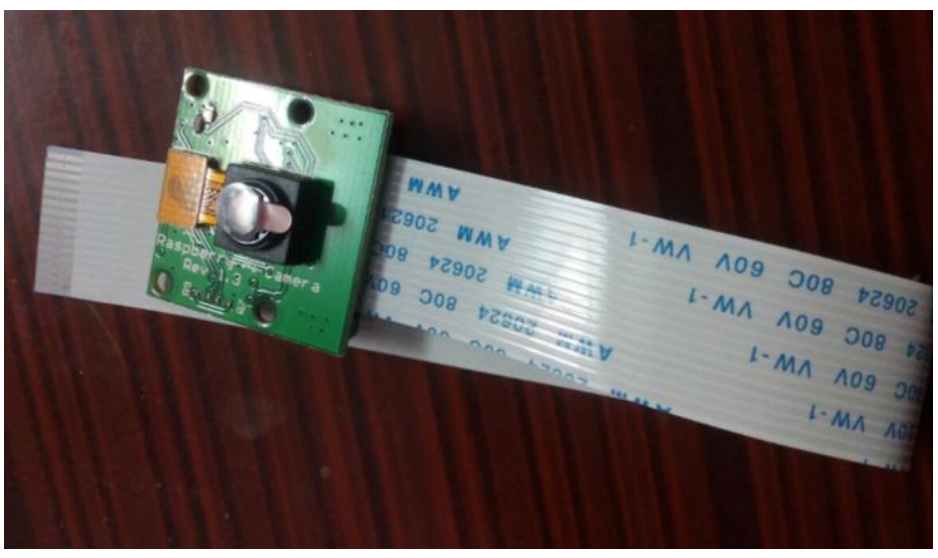

Figure 3. Raspberry pi camera module V2 
Raspberry Pi is a small single-board computer developed by Raspberry Pi foundation, in the United Kingdom, for teaching purposes and DIY projects. Its 1.2 GHz ARM Cortex processor and 1 GB RAM make it suitable for the project [7]. As it has On-board Bluetooth and Wi-Fi, so connecting the device to the internet is also very convenient. Over the years, Raspberry Pi foundation has released several version of this On-board computer. We employed Raspberry Pi model 3 B. It works as the main brain of our system and fulfills all the computational requirements for this project. The main advantage is its small size, low cost, and good computational power.

For displaying the data, a simple LED display was employed. The partially reflective twoway mirror helps our device to mimic more like a usual mirror. 5 Megapixel Raspberry Pi Camera Module V2 is used for capturing and detecting the user. For sound input and output, a simple USB microphone and a $3.5 \mathrm{~mm}$ jack Speaker are used respectively. For controlling the AC home appliance an SPDT relay board is used.

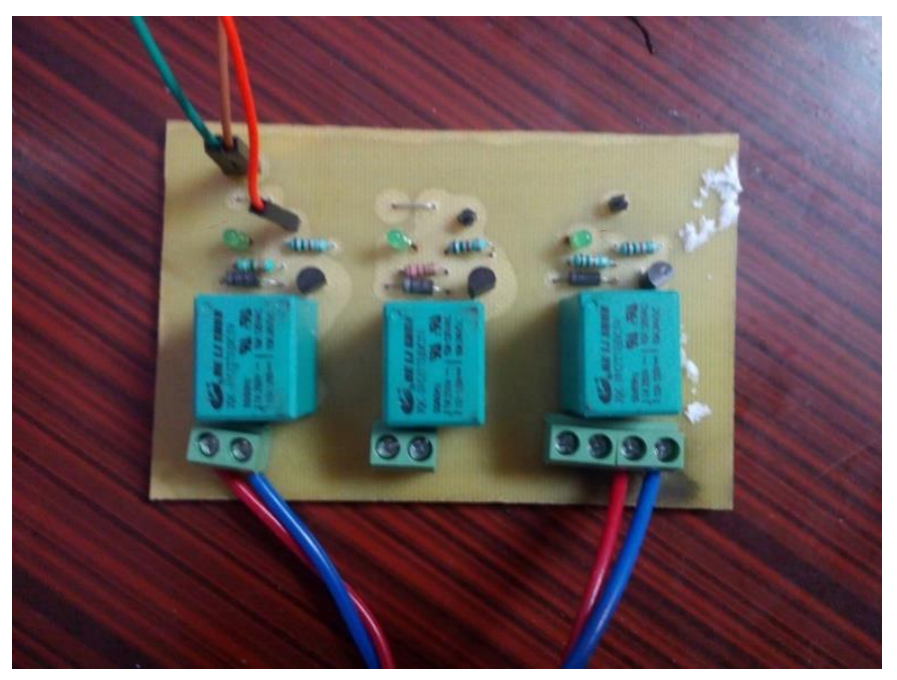

Figure 4. Relay module

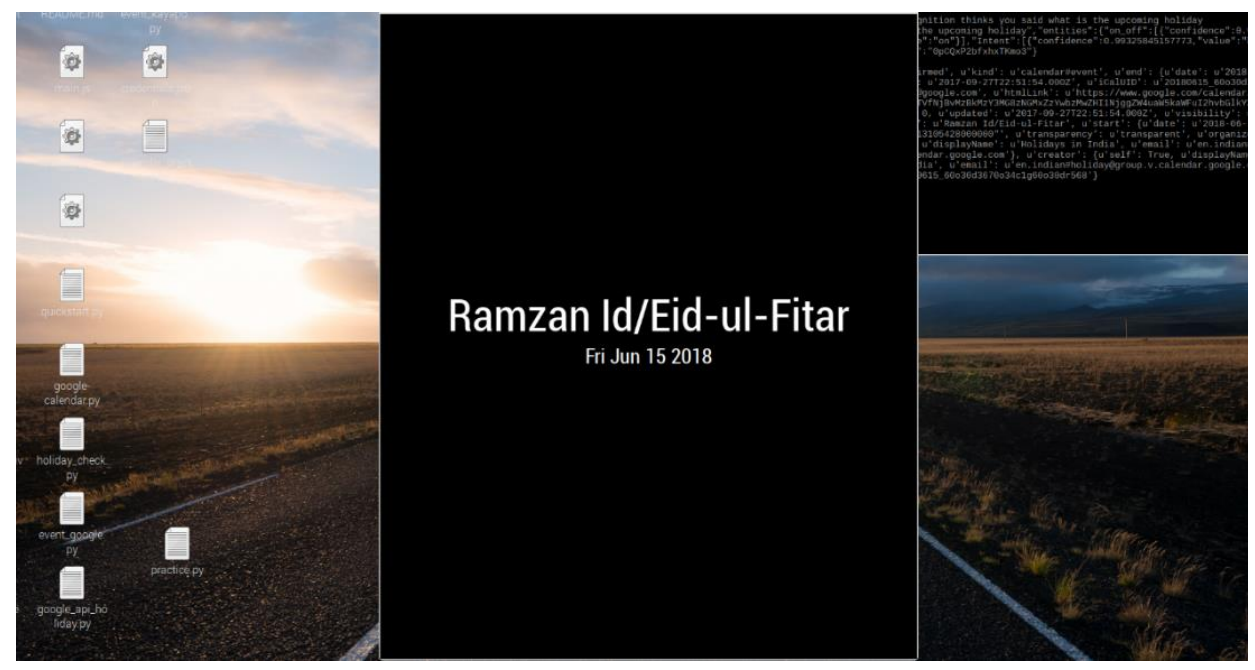

Figure 5. Mirror gui showing upcoming holiday 
After hardware overview, now the software description is covered in this phase. Raspberry Pi needs an operating system which is stored in a separate SD Card. We used the Raspbian 9 (stretch) operating system recommended for Raspberry Pi. It is an open-source Debian based operating system [8] which is free and easily customizable. It provides a stable and fast environment for our mirror. With over 35,000 packages, it is suitable for our project.

For programming purposes, we used Python programming language. It is a high-level language with very easy syntax. It has a large number of speech and image processing packages suitable for our system. OpenCV (Open Source Computer Vision) [1] library is used for face detection. It can easily be installed as python module.

\subsection{Workflow}

The basic workflow of the system is shown in [Figure 6].

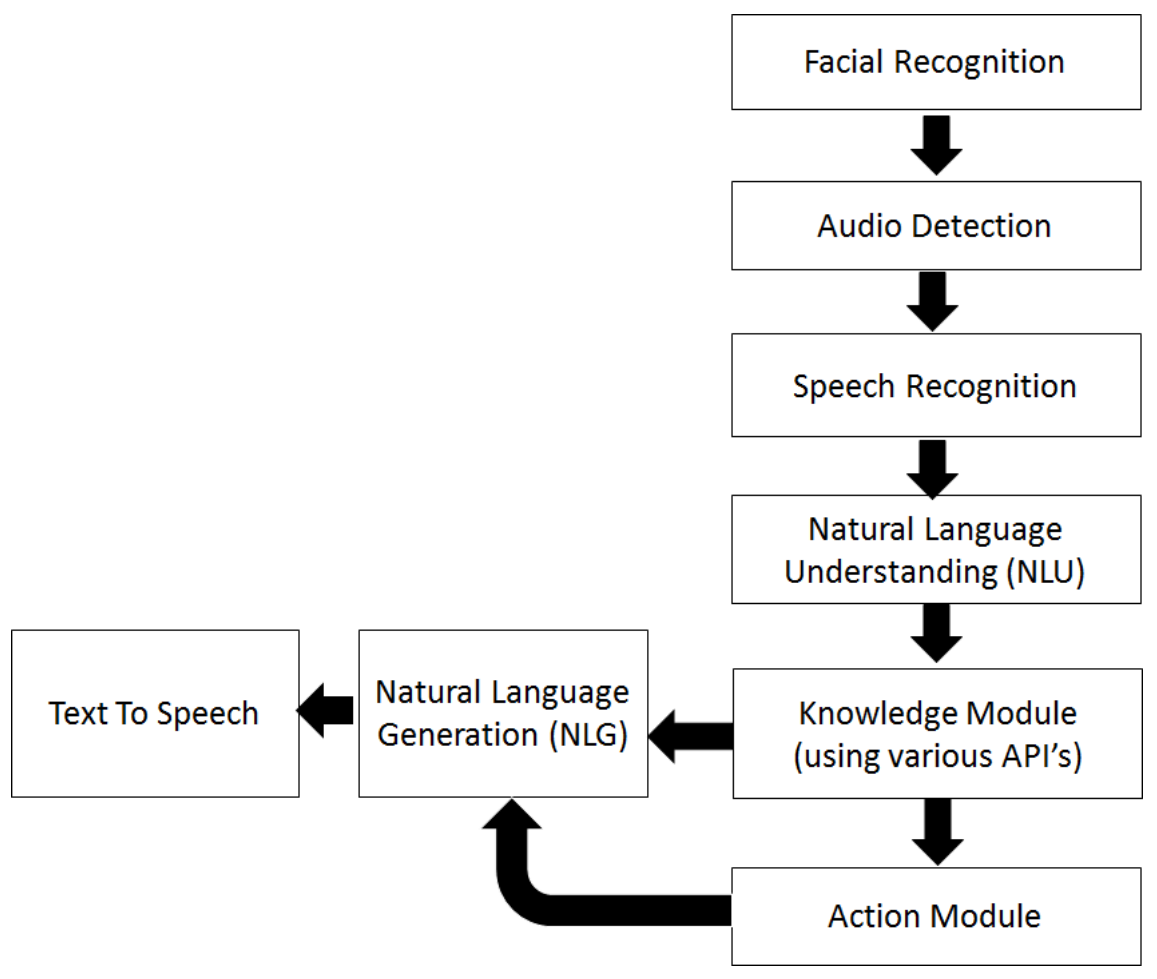

Figure 6. Workflow diagram of the mirror

The simple working of the system is analogous to the conversation between two people. One person tells something to the other and the other will respond and work according to the instructions given by the first.

Likewise, when this device detects any face in front of it, it starts listening for the instructions. For detecting the face, it uses OpenCV module of python after capturing an image through the camera. As the person standing in front of the mirror gives the instruction, the mirror detects it as an audio using a microphone. Processing audio directly for further modules is not as fast as processing the text. So the audio is converted into text using Google Speech Recognition module. 
The converted text is in the form of the sentence. To figure out what that sentence actually means, an NLU (Natural Language Understanding) system is used. NLU system extracts intents and entities from the text so that the device can understand what the user actually means.

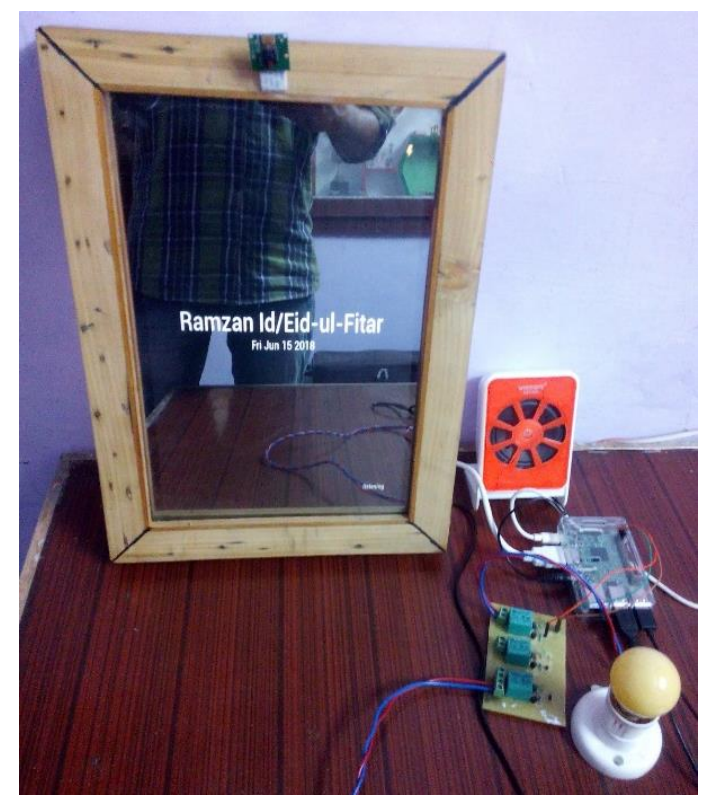

Figure 7. Final setup of witmirror showing upcoming holiday

Now, this intent is passed to the knowledge module. Knowledge module is used to connect the device with various APIs available on the Internet. It basically works as data extractor, which extracts the data according to the intent provided by NLU system. Further, if the device is instructed to perform certain tasks, that does not need any data extraction from the internet, then knowledge module calls the Action Module to perform that particular task. For example, if the user instructs the device to Switch On the lights, Knowledge Module does not need to extract any data from the internet. It just has to perform the action (here, turning on the light) and show the result on the display.

Now, as each result is shown on the display as a sentence, so the device has to convert the data given by the Knowledge Module to the natural sentence that makes sense. For that purpose, Natural Language Generation (NLG) system is used. It takes the data as input and converts it into language sentence similar to that spoken by humans. Apart from displaying the generated sentence, it is also spoken out by the mirror through speakers. For that Google Text To Speech Converter is used, which creates the audio.

\section{Conclusion \& future scope}

As proposed, the device is successfully designed which fulfilled all the technical objectives and yet looked like a simple wall-mounted mirror. The spectacular thing about this project is its allured features and simple look. The device is successful in interpreting the instructions and giving the desired output. Of course, there are some limitations identified during the development of the project. If the internet connection is slow, the device takes more time for processing as the NLU system is internet-based. Moreover, extraction of data also becomes slow with poor connectivity. Yet, the GUI environment is quite fast as it uses pure Python GUI and does not depend on heavy frameworks like node.js. 
We hope that this research will be beneficial for the researchers who are interested in similar topics. In future, more features can be added to the device. Touchscreen based devices can also be made, which can ease the level of interaction with the device. By connecting the system to the API of any search engine, the mirror can become the hub for a large bunch of data accessible in quite an interactive way.

\section{References}

[1] Tatiana Lashina, Intelligent bathroom, "In European Symposium on Ambient Intelligence," EUSAI'04, Eindhoven, Netherlands, (2004)

[2] Alpay Kasal and Sam Ewen, "A project of interactive mirror with artsy visuals in Lit Studios," (2008)

[3] T Lakshmanan, "Two-way mirror to allow the LEDs and to display information on screen," Chalmers University of Technology in Sweden, (2009)

[4] New York Times Research and Development Lab, "A Magic mirror using Microsoft Kinect to track movements," (2011)

[5] Jose and Jane et al., "Home automated smart mirror as An Internet of Things (IoT) implementation," IJARCCE vol.6, no.2, (2017)

[6] E. Iwabuchi, M. Nakagawa, and I. Siio. "Smart makeup mirror: Computer-augmented mirror to aid makeup application," $13^{\text {th }}$ International Conference on Human-Computer Interaction, Part IV: Interacting in Various Application Domains, pp.495-503, Springer, (2009) DOI: 10.1007/978-3-642-02583-9_54

[7] Piyush Maheshwari, Maninder Jeet Kaur, and Sarthak Anand, "Smart Mirror: A reflective interface to maximize productivity," International Journal of Computer Application, vol.166, no.9, pp.30-35, (2017)

[8] Khanna V., Vardhan Y., Nair D., Pannu P, "Design and development of a smart mirror using raspberry Pi," International Journal of Electrical, Electronics and Data Communication, vol.5, no.1, pp.63-65, (2017)

[9] ERCIM Working Group SESAMI, "Smart Environments and Systems for Ambient Intelligence”, (2006)

[10] H. Tokuda, "Smart furniture: A platform for creating context-aware ubiquitous applications everywhere," in Proceedings of the International Conference on Embedded and Ubiquitous Computing, pp.1112, (2004) DOI: 10.1007/978-3-540-30121-9_108

[11] Derrick Gold, David Sollinger, and Indratmo., "Smart reflect: A modular smart mirror application platform," IEEE $7^{\text {th }}$ Annual Information Technology, Electronics and Mobile Communication Conference, pp.1-4, (2016) DOI: $10.1109 /$ IEMCON.2016.7746277

[12] K. Ashton, “That 'Internet of Things' Thing,” RFID Journal, Jun, (2009)

[13] Z. Pang, "Technologies and architectures of the Internet-of-Things (IoT) for health and well being," PhD Thesis, Royal Institute of Technology (KTH), Stockholm, Sweden, (2013)

[14] E. Aarts, H. Harwig, and M. Schuurmans., "Ambient intelligence, the invisible future," McGraw Hill, New York, pp.235-250, (2001)

[15] G. Yolcu, S. Kazan and C. Oz, "Real time virtual mirror using Kinect," Balkan Journal of Electrical \& Computer Engineering, vol.2, no.2, June, (2014)

[16] https://github.com/HackerShackOfficial/AI-Smart-Mirror/ 